\title{
Las haciendas cañeras en el sur del lago de Maracaibo-Venezuela (siglos XVI-XVII)
}

por

\author{
Luis Alberto Ramírez Méndez ${ }^{1}$
}

Universidad de Los Andes, Mérida-Venezuela

En el presente análisis se estudian las haciendas cañameleras ubicadas al sur del lago de Maracaibo, área jurisdiccional de Mérida (Venezuela), donde se desarrolló el cultivo y procesamiento de la caña de azúcar a partir del siglo XVI. Es un estudio cuantitativo y cualitativo sobre el origen de las haciendas de caña, su evolución y producción, su infraestructura y rentabilidad. La investigación se asienta en las fuentes documentales inéditas existentes en los archivos nacionales y extranjeros.

PALABRAS ClAVE: haciendas; cañaverales; trapiche; miel; melote; azúcar.

\section{INTRODUCCIÓN}

El estudio de las haciendas en Hispanoamérica ha atraído la atención de numerosos analistas a partir de $1950^{2}$. Desde esa fecha han variado significativamente los enfoques aplicados a la indagación de esta interesante temática durante el período colonial y postcolonial. Los análisis se han centrado en diversos aspectos como la evolución y conformación de la propiedad, los propietarios y sus familias, los medios y útiles de producción, capital, mano de obra, trabajo, tecnología, comercialización, productos, sociabilidad, administración, productividad, valor, infraestructura, viabilidad, costos y precios,

${ }^{1}$ Doctor en Historia, profesor miembro del grupo investigación de Geografía Histórica de las Regiones Americanas, (GHIRA) y profesor en la Maestría en Historia de la Escuela de Historia, Facultad de Humanidades y Educación, Universidad de Los Andes, Mérida-Venezuela.

2 Borach, 1951: 20-49. Chevalier, 1952. 
tanto de los inmuebles como de los beneficios, en diversas regiones del Nuevo Mundo, precisando las variaciones que experimentaron en cada espacio geográfico, fundamentalmente en la Nueva España ${ }^{3}$ y Perú $^{4}$, en donde se realizaron los estudios pioneros y posteriormente en Bolivia ${ }^{5}$, Argentina ${ }^{6}$ y la Nueva Granada? .

En Venezuela, se han efectuado aportes sobre esta temática, con la publicación, encabezada por Arcila Farías, que proporcionó un avance hacia el conocimiento y tipificación de la hacienda cacaotera en la provincia de Venezuela ${ }^{8}$; pero es necesario puntualizar que ese enfoque está apegado a la visión marxista, similar al utilizado por Pablo Macera en su estudio sobre las haciendas peruanas, ambos investigadores parten del supuesto que las haciendas se formaron y desarrollaron a partir del traslado y funcionamiento de las estructuras feudales al Nuevo Mundo, lo que revela incoherencias entre el planteamiento teórico y la realidad estudiada. Otros autores, han centrado sus estudios sobre el origen, evolución y desarrollo de haciendas azucareras en el estado de Aragua $^{9}$ y sobre las haciendas llaneras jesuíticas inmediatas a los ríos Casanare, Meta y Orinoco ${ }^{10}$.

En la región merideña, se destacan los estudios de Samudio sobre las haciendas de los jesuitas tanto en los valles altos inter-montanos de la serranía como de las cacaoteras del sur del Lago de Maracaibo ${ }^{11}$. Entre tanto, otros investigadores indagan las haciendas ubicadas en el curso del río Chama medio $^{12}$, las de tabaco en Barinas ${ }^{13}$. Por su parte, Peter Linder aborda las relaciones de producción en el sur del Lago de Maracaibo a finales del siglo XIX y principios del $\mathrm{XX}^{14}$.

En torno a la definición de hacienda existe consenso entre diversos autores, al considerar la proposición de Wolf y Mintz, quienes la han conceptuado

3 Riley, 1979: 49-69 y 242-272. Taylor, 1979: 71-102. Brading, 1979: 49-69. Tovar Pinzón, 1979: 132-241. Bazant, 1975. Lindley, 1987. Macera, 2 (Hungría, 1971): 34-86. Lavrin, XXXV/97 (México, 1975): 75-116. González, 1969. Follet, LXV/233 (Sevilla, 2005).

4 Mörner, 1979: 316-396. Lockart, 1982, 20-48. Macera, t. XXX (Szeccd, 1971): 3-43.

5 Jackson y Gordillo Claure, LIII/199 (Sevilla, 1993): 723-759.

${ }^{6}$ Birrocco, LIII/1 (Sevilla, 1996):1-26. Franklin, 1992, t. I y II.

7 Villamarín, 1979: 145-160. Colmenares, 1969. Tovar, 2007: 101-114. Escorcia, 10 (Bogotá, 1982): 119-137.

8 Arcila, 1968.

9 Tavera, 1995. Banko: LXXXVIII/352 (Caracas, 2005a): 157-179.

10 Samudio, 1993.

11 Samudio, 1985; 2003, t. I.

12 Tallafero, 1979. Gelambi, 1979. Espinoza, 1980. Suárez, 1984.

13 Ruiz, 2000.

14 Linder, 19/V/5 (Caracas, 1987): 283-291. 
como «una propiedad agrícola operada por un terrateniente, quien dirige una fuerza de trabajo subordinado organizada para aprovisionar a un mercado reducido, con la ayuda de un pequeño capital» ${ }^{15}$. Además, se ha aceptado que el concepto es tan sólo un polo en un continuum de variaciones del mismo fenómeno ${ }^{16}$.

En el presente estudio se analizan las haciendas cañameleras ubicadas en la planicie sur del lago de Maracaibo, las que tempranamente se desarrollaron y posibilitaron el ingente desarrollo de la economía emeritense en los siglos XVI y XVII. El área estudiada se extiende entre Estanques y la desembocadura del río Escalante, y su vértice en el río Pocó, que escurre en el actual límite entre los Estados Mérida y Trujillo. La investigación se centra en el estudio cualitativo de la infraestructura y cuantitativo de la producción.

La investigación se fundamenta en las fuentes documentales inéditas existentes en el Archivo General de la Nación de Santa Fe de Bogotá (Colombia) y el Archivo General del Estado Mérida (Mérida-Venezuela), donde existen series documentales que se refieren al sur del Lago de Maracaibo como las enajenaciones de propiedad, inventarios de bienes, valuación, peritajes y poderes, los que posibilitan conocer el proceso productivo de las haciendas. La información es tabulada y procesada y se presentan los resultados.

\section{Origen y EVOLUCiÓn de las haciendas CAÑERAS EN EL SUR DEL LaGo DE MaracAibo}

El proceso formativo de las haciendas, ha reconocido su base en las estancias, las que han sido poco estudiadas, sobre la evolución de las mismas en la Nueva Granada, Torres, ubica su surgimiento a finales del siglo XVI, definiéndolas como centros de producción agrícola y ganadera, explotadas bajo el modelo de «tierras de labor» característico de la meseta castellana, constituyendo propiedades asignadas a los primigenios encomenderos, quienes utilizaron los beneficios obtenidos del tributo indígena para invertirlo en sistemas de siembra; considerándolas como unidades productivas con aportes de trabajo familiar, previas a la aparición de las haciendas ${ }^{17}$ y las clasifica como estancias de ganado mayor. En Mérida, a diferencia de lo ocurrido en Susa, se utilizó directamente el trabajo aborigen mediante la

\footnotetext{
15 Wolf y Mintz, 1979: 493-591.

16 Idem.

17 Torres, 2003: 227-228.
} 
prestación de servicios personales para establecer las roturaciones y los cultivos iniciales.

A diferencia de lo ocurrido en Mérida, Taylor explica que en Oaxaca en la Nueva España, no fue sino a principios del siglo XVII, cuando comenzaron a desarrollarse unidades de producción denominadas «haciendas», para designar una propiedad más compleja que la estancia, entendida como «una nueva entidad económica dedicada a abastecer mercados locales tanto de productos animales como granos» ${ }^{18}$. Macera, explica que una situación similar sucedió en el ande peruano en la formación de las primeras haciendas mediante la enajenación de fanegadas de tierra de los indígenas a los blancos ${ }^{19}$. En la Nueva Granada, Tovar afirma que las haciendas se desarrollaron a lo largo de dos centurias a partir del el siglo XVI, en la medida que se incorporaron tierras y se expandió la frontera agrícola ${ }^{20}$. El desarrollo de las mismas estuvo orientado a atender la demanda de sectores urbanos y mineros, lo cual no significó la ausencia de una demanda rural, debido a la diversificación de la producción en el ámbito interno posibilitando abastecer de variados productos $^{21}$ a diferentes mercados y a distintos consumidores.

Asimismo, la conformación de las haciendas trajo aparejado el establecimiento de una compleja red de relaciones dirigidas a la vinculación y control de los mercados donde se comercializaban sus productos ${ }^{22}$. Esos enlaces se basaban en la expresa intención de los propietarios vinculados mediante parentesco o bien de las instituciones eclesiásticas en realizar adquisiciones simultáneas de diferentes haciendas ubicadas en desiguales pisos altitudinales, lo que les facilitaba realizar cultivos en heterogéneas condiciones climáticas y edáficas. La diversidad de producción obtenida en esos predios fue complementaria entre sí, conformándose así una red subsidiara de abastecimiento y consumo ${ }^{23}$. Además, se crearon conexiones comerciales entre los centros de acopio y distribución, donde se embarcaban, remitían, recibían, fletaban, disponían de medios de transporte y se mantenían agentes autorizados y apoderados para ejercer la función mercantil. De esa forma, los hacendados cumplían, alternativa y simultáneamente, las funciones de cosecheros y comerciantes, remitiendo productos agrícolas e im-

\footnotetext{
18 Taylor, 1979: 77.

19 Macera, XXX/IV (Hungría Szeccd, 1971): 5.

20 Tovar, 2007: 103.

21 Ibidem: 105.

22 Ibidem: 103.

23 Idem. Samudio, 1985: 33-41. Ramírez, 2005: 321-393.
} 
portando mercaderías manufacturadas de los centros de producción a los de consumo subsidiario ${ }^{24}$.

En cuanto a la mano de obra, existe consenso en los autores sobre la diversidad de grupos étnicos empleados en las haciendas. Mientras en la Nueva España ${ }^{25}$ y en Perú ${ }^{26}$ fueron mayoritariamente indígenas, en la Nueva Granada coexistieron tanto amerindios con mestizos ${ }^{27}$. En Venezuela, fueron fundamentalmente de origen africano ${ }^{28}$, aunque es preciso señalar que no existió una absoluta diferenciación entre los diversos grupos étnicos que laboraban en las haciendas, donde coexistieron trabajadores de diversas etnias. En cuanto a los sistemas de trabajo a los que se sometieron los trabajadores, fueron también diversos como la encomienda ${ }^{29}$, la mita, el cuatequil ${ }^{30}$, el concertaje ${ }^{31}$ y la esclavitud.

En relación al capital empleado en las haciendas, éste tuvo un origen disímil. En algunas ocasiones, fue obtenido de las actividades mineras; en los casos donde las haciendas surgieron como centros de abastecimiento de economías basadas en la explotación de los metales. En otros, representaron las crecientes inversiones de estirpes unidas a través de la política de enlaces matrimoniales ${ }^{32}$, entregados mediante las $\operatorname{dotes}^{33}$ y trasmitidas por herencias y donaciones en sociedades endogámicas ${ }^{34}$. En general, se recurrió a la obtención del crédito al sistema financiero eclesiástico a través de la suscripción de $\operatorname{censos}^{35}$. Adicionalmente, hubo sectores privilegiados que contaron con elevadas sumas de efectivo como lo fueron las órdenes eclesiásticas, especialmente los jesuitas ${ }^{36}$ y las mendicantes femeninas, que dis-

24 Kickza, 1986: 163-202. Tovar, 2007: 105. Stanley y Stein, 1975: 151. Samudio, 2003: 145-233.

25 Taylor, 1979: 90-91. Riley, 1979: 60-64.

26 Lockhart, 1982: 278-290.

27 Tovar, 2007: 105-106.

28 Maza, 1968: 104. Samudio, (Caracas, 1998): 451-548. Colmenares, 1979: 161-162.

29 Riley, 1979: 51-60. Konetzke, 1977: 160-181. Colmenares, 1977: 161-187 y 156-167.

30 Konetzke, 1977: 181-189.

31 Ibidem: 189-191.

32 Kicza, 1986: 177-202. Socolow, 1991: 39-40. Langue: 2000: 69-81.

33 Siegrist y Samudio, 2006.

34 Arango, 2003: 24 y 46-49.

35 Lavrin, XLVI/4 (Duke, 1966): 372-393; 53/1 (Duke, 1993): 27-49. Bauer, 63/4 (Duke, 1983): 711-740; 1986. Jiménez, 71/3 (Duke, 1991): 501-529. Martínez, 1995. Von Wobeser, 1994. Ferreira, 1995: 38-78. Langer y Hames, 74/2 (Duke, 1994): 285. Andrein, 13/1 (Cambrige, 1981): 1-19. Troconis, 1971; 1982. Terán, II semestre/1 (Quito, 1991): 22-48. Quiroz, 1/50 (Duke, 1994): 194-229. Marmolejo y Tablante, 1984.

36 Samudio, 2003: 195-201. Tovar, 2007: 104. 
pusieron de ingentes cantidades de circulante para ser invertidas en el sistema productivo de las haciendas ${ }^{37}$. Finalmente, en cuanto a la rentabilidad de las mismas, hay heterogeneidad de opiniones en los autores. Inicialmente, se había aceptado que había un $5 \%$ de retorno en las grandes haciendas ${ }^{38}$, pero se ha demostrado que en 20 ó 40 años los índices de riqueza se multiplicaban por 5 y 10 veces $^{39}$.

\section{LAS HACIENDAS CAÑAMELERAS}

Uno de los dos rubros agrícolas de importancia que se desarrollaron en la planicie lacustre fue el cultivo de la caña de azúcar, el que ha sido objeto de profusos estudios en América ${ }^{40}$, los que comprenden diversos tópicos, desde su introducción al Nuevo Mundo ${ }^{41}$, su procesamiento para obtener la miel, el melote y el azúcar, las técnicas aplicadas que abarcan en su etapa artesanal, las labores de trapiche, sus avances tecnológicos, sus sistemas de trabajo, trabajadores y su evolución hasta la introducción de las modernas procesadoras industriales y la conformación de los centrales azucareros ${ }^{42}$.

La gramínea fue traída a las Antillas a principios del siglo XVI. En ese sentido, se afirma que Colón trasladó las primeras cañas a La Española en 1501. Entre tanto, en la Nueva Granada, se atestigua que Pedro de Heredia la transportó a Cartagena de Indias hacia 1538, también, se sostiene que Sebastián de Belalcázar la trajo porque se hacía referencia a su cultivo en Cali en $1548^{43}$. Desde aquellas ciudades se diseminó a través de la ruta del Magdalena hasta Mérida, donde se refiere su cultivo en 1581 y la construcción de trapiches ${ }^{44}$. A principios del siglo XVII, los cañamelares se expandían en la cuenca del Chama, conformándose haciendas trapicheras.

37 Ramírez, 2005: 397-442.

38 Ruiz, 2000: 270-272.

39 Tovar, 2007: 106.

40 Santamaría y García, LXV/233 (Sevilla, 2005): 9-32.

41 Barret y Schuartz, 1979: 532-571. Criton, 1979: 573-608. Ramos Gómez, LXV/233 (Sevilla, 2005): 49-78. Shuartz: LXV/233 (Sevilla, 2005): 79-116. Barret, 1970.

42 Klaren, LXV/233 (Sevilla, 2005): 32-34. Sánchez, LXV/233 (Sevilla, 2005): 147-172. García, LXV/233 (Sevilla, 2005): 173-192. Moreno, 1978. Sandoval, 1951. Banko, 91/Año 23/XXIII (Caracas, 2005b): 341-360; XXXVIII/352 (Caracas, octubre-diciembre, 2005a): 157-179. Molina, 13 (Caracas, 2005): 199-224. Rodríguez, 2008.

43 Ramos Gómez, LXV/233 (Sevilla, 2005): 49-50.

44 En 1581 Martín Pujol se obligó a «hazer labranzas de cañaverales... y si fuere necesario hazer algún trapiche». Millares, 1966: 47. 
En el sur del lago de Maracaibo se describen los cultivos de caña en $1607^{45}$ y se situaban en los valles de Chama ${ }^{46}$, Bobures, Espíritu Santo ${ }^{47}$, Castro, Mojaján, Chirurít ${ }^{48}$ y Arapuey, espacios donde fueron una actividad secundaria en relación al cultivo del cacao, debido a que sus productos se requerían para la preparación del chocolate y otras delicias culinarias.

La tecnología en el cultivo y procesamiento de la caña de azúcar se diferenció notablemente de la utilizada en las haciendas cacaoteras, porque se hizo necesario la roza total, eliminando la selva tropical para dar paso a los cañaverales, en espacios definidos a los que se denominó «suertes o tablones» ${ }^{49}$. El cultivo se iniciaba con el arado y roturación del suelo, lo cual se hacía con rejas, para desmenuzar la tierra; luego se rastrillaba con el fin de romper los terrones más grandes que dejaba el proceso de arado. Inmediatamente, se procedía a nivelar los tablones dejando un ligero declive que permitiera escurrir el regadío que se transportaba a través de canales y acequias. Sucesivamente, se rompían las capas subyacentes de la tierra que el arado no había alcanzado y por último se surcaba para iniciar la siembra ${ }^{50}$.

La siembra consistía en soterrar los esquejes de caña, generalmente de una dimensión de tres yemas, con sumo cuidado para evitar obstáculos en su proceso de crecimiento. Seguidamente se atendía al regadío permitiendo el paso de las primeras corrientes de agua, las que se le proporcionaban inmediatamente después de la siembra, pero evitando el arrastre de la materia orgánica, facilitando con ello el crecimiento de los brotes. En la irrigación de los cultivos cañeros se acudió al sistema de acequias y esclusas para dirigir el vital líquido a través de las distintas haciendas, manteniendo de esa forma la humedad necesaria, impidiendo la anegación de los sembradíos y cuidando la sequedad requerida para el período de cosecha. Ocasionalmente, se perdie-

45 Diego de Villanueva y Gibaja, expresaba en 1607, que Gibraltar tenía «en su comarca muchas tierras fértiles donde se da el algodón, maíz, cacao, caña dulce y otras cosas». Relación Geográfica, 1964: 207.

46 Testamento de Antonio Reinoso, Mérida, 1 de marzo de 1658, Archivo General del Estado Mérida (AGEM), Protocolos t. XXIV, folios 10r-17v.

47 Carta de dote de Inés Plaza, Mérida, 9 de febrero de 1669, AGEM, Protocolos, t. XXVIII, folios 10r-19v.

48 Inventario de la hacienda de Chirurí, Valle de Chirurí, 16 de noviembre de 1644, AGEM, Mortuorias t. IV, Mortuoria de Joseph Rodríguez Melo, f. 168r.

49 «...dos tablones y suertes de caña dulce que se muele entre el año», Inventario de la hacienda de Chirurí, Valle de Chirurí, 16 de noviembre de 1644, AGEM, Mortuorias t. IV, Mortuoria de Joseph Rodríguez Melo, f. 168r.

50 Suárez, 2001: 150-151. 
ron los cultivos en suertes de caña, debido a largas temporadas de sequías ${ }^{51}$, ocasionando la ruina de los cañaverales e intentos de resiembro ${ }^{52}$.

Periódicamente, se realizaban las limpias con el concurso indígena encomendado ${ }^{53}$, luego se podaban los brotes superfluos de la planta utilizando los azadones, las palas y las paletillas ${ }^{54}$. Finalmente, la caña se cosechaba después de diez o doce meses de sembrada, cuando los tallos estaban más jugosos, desplegando el proceso de zafra, en cuyas faenas se empleaba el machete. En la zafra, los, mayordomos fueron cuidadosos, debido a que la caña debe ser cosechada antes de espigar, cuando su rendimiento es óptimo y se denominaba «caña hecha» ${ }^{55}$. Por el contrario, si se procedía a realizar la siega sin atender a tan precisión, se perdía la cosecha porque los tallos carecen del suficiente jugo y sacarosa para solidificar la panela y obtener el azúcar, por consiguiente se desperdiciaban esfuerzos en fomentar los cañaverales $^{56}$.

51 «...esto abrá veinte días que lluebe gota con que están los caminos más que buenos y es todo hecho una yesca, y la caña que sembré perdida sírvase Dios con todo», Carta de Salvador de Trejo a Ignacio de Trejo, Gibraltar, 16 de diciembre de 1662, AGEM, Asuntos Diversos t. VIII, 1664, n. ${ }^{\circ}$ 3, folios 197r-198v.

52 «...en este dicho año de seiscientos sesenta y tres, no ubo mas caña hecha que poder moler sólo estos dos pedazos, las quales estuvieron tan fallados y tan ruynsita la caña que fue suerte que se hicieran los dichos ciento ochenta y quatro panes de asúcar en este presente año de sesenta y cuatro», Libro de cuentas de la hacienda de Torondoy. 1664, ibidem, f. 115v.

53 «...y así se lo a de advertir vuestra merced al casique de todos los demás: les advertirá vuestra merced me acudan con toda voluntad, y que estén todos juntos sin faltar ninguno otro día, después de pascua, y en el inter que yo boy an deshervado los cañaverales y todas las huertas», Carta de Salvador de Trejo a Ignacio de Trejo, Gibraltar, 16 de diciembre de 1662, ibidem, folios 197r-198v.

54 «Más dose pesos que se gastaron en calsar hachas y paletillas y asadones de dicha asienda para el beneficio de ella y algunas paletillas que se compraron nuebas = no pongo en esta quenta los tasises y machetes que hecho nuebos, pues esos aunque han sido menester para la hacienda se los he dado a mi gente para que trabajasen con ellos así no los cargo en esta quenta», Libro de cuentas de la hacienda de Torondoy, 1664, ibidem, f. 120r.

55 «... y se empessó la molienda entonses por no aver avido antes caña echa para moler y se comensó de un pedazo de caña que está tras la casa de las gallinas, a espaldas de la casa grande y molino por dicho mes de febrero», Libro de cuentas de la hacienda de Torondoy, 1664, ibidem, f. 114r.

56 «El cañaveral de la loma por donde vuestra merced salió, que lo dejó vuestra merced ya perdido y no lo ygnora vuestra merced la prueba la bes si quajaba siquiera papelones y no fue posible con he aprovechado la poca y ruin caña que tenía en su sembrar otros cañaverales y no le volveré a beneficiar porque es que se trabaja en ellos de balde y sin provecho», Carta de Salvador de Trejo a Ignacio de Trejo, Torondoy, 15 de junio de 1663, ibidem, folios 201r-202v. 
Los tablones se diferenciaban por su edad ${ }^{57}$ y tamaño permitiendo diferentes zafras en distintas épocas. Así se obtenía caña para mantener activos los trapiches durante todo el año. A aquellas suertes que habían recibido $\operatorname{cortes}^{58}$ de primera, segunda y tercera vez, se las denominó respectivamente siembra, soca y resoca ${ }^{59}$. Luego se evaluaban los cañaverales de resoca para determinar los que requerían de nuevos resiembros a efectos de mantener las suertes productivas ${ }^{60}$.

El procesamiento de la caña se realizaba en la casa de molienda, edificada sobre horcones, techados de palma ${ }^{61}$, donde se cimentaban los trapiches, los cuales fueron una notable innovación tecnológica que se alcanzó en los ingenios de La Española, y que rápidamente se difundió en el continente ${ }^{62}$ impulsados por tracción de agua y/o sangre ${ }^{63}$, en el que se instalaban tres piedras o masas moledoras, verticales ${ }^{64}$, que giraban me-

57 En el inventario de la hacienda de José de Cepeda y Santa Cruz se hizo constar: «ytem más onse tablones de caña de buen tamaño los tres, digo, quatro biexos y los siete nuevos», Inventario de José de Cepeda y Santa Cruz, San Antonio de Gibraltar, 21 de abril de 1661, Archivo General de la Nación, Bogotá (AGNB), Real Audiencia de Bolívar y Venezuela, S.C, 50,1 , Doc. 1 , f. $26 \mathrm{v}$.

58 «...ocho suertes de caña, dos de ellas de caña pequeña para moler y las cuatro de caña más mediana y los dos tablones de ella mayores que los seis», Inventario de la estancia de la Sabana del Espíritu Santo, La Sabana del Espíritu Santo, 28 de enero de 1666, AGEM, Mortuorias t. VIII, Mortuoria de Juan de Sologuren, folios 11v-14r.

59 Samudio, 1985: 87.

60 «...pues allé los cañaverales perdidos y los tengo resembrados, o por menor decir, sembrados de nuevo con en esa ocupación y deshierbos», Carta de Salvador de Trejo a Ignacio de Trejo, Gibraltar, 16 de diciembre de 1662, AGEM, Asuntos Diversos t. VIII, 1664,

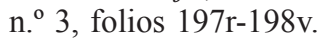

61 «...una casa de horcones cubierta de palma, por un costado ya vencida y los maderos de aliñar podridos y dentro un trapiche», Inventario de la hacienda de la Sabana de Domingo de Plaza. La Sabana del Espíritu Santo, 30 de septiembre de 1669, AGEM, Mortuorias t. XI, Mortuoria de Domingo de Plaza, folios 10v-11r.

62 Rodríguez Morel, 1992: 94.

63 «...nuebe bestias mulares, cuatro mulas y cinco machos herrados con el hierro del dicho difunto. Ytem más tres caballos el huno de camino y los dos de moles y cargar», Inventario de José de Cepeda y Santa Cruz, San Antonio de Gibraltar, 21 de abril de 1661, AGNB, Real Audiencia de Bolívar y Venezuela, S.C, 50, 1, Doc. 1, f. 26r-v.

64 «...una casa grande donde está un trapiche de tres masas de bera viejo y maltratado», Inventario de la hacienda de Chirurí, Valle de Chirurí, 16 de noviembre de 1644, AGEM, Mortuorias t. IV, Mortuoria de Joseph Rodríguez Melo, f. 168r. 
diante un mecanismos de engranajes y cadenas ${ }^{65}$, lubricadas con sebo ${ }^{66}$ para evitar que se atascaran. Las masas de piedra eran elaboradas por los maestros canteros, mientras las de madera las fabricaban los carpinteros; cuya técnica de comprobada eficacia y ahorro de trabajo, determinó su expansión ${ }^{67}$.

Las labores exigían un tren de tres, cinco o más fondos de cobre ${ }^{68}$, los que proporcionaban eficiencia en la cadena de calentamiento, clarificación, evaporación y concentración. Las pailas se fabricaban fundidas o martilladas ${ }^{69}$, y se adquirían en el puerto ${ }^{70}$. Los fondos se colocaban sobre las hornallas ${ }^{71}$, construidas con ladrillos, unidos con barro y melaza, cuyo mortero se solidificaba con el calor. Asimismo, los fogones se comunicaban con una alta chi-

65 «...de que se ase asúcar una casa de trapiche con un con un moxicón un par de cadenas de respeto». Carta de arrendamiento, Torondoy, 4 de diciembre de 1662, AGEM, Asuntos Diversos t. VIII, 1664, n. $^{\circ} 3$, folios $85 \mathrm{r}-87 \mathrm{r}$.

66 «Más media arroba de sevo para untar los dientes y golletes del trapiche ha costado todo seis reales», Libro de cuentas de la hacienda de Torondoy, 1664, ibidem, f. 119v.

67 Ramos Gómez, LXV/233 (Sevilla, 2005): 54.

68 «...tres fondos asentados en las hornallas de cobre batidos, la huna que hiso veinte y ocho votixas de agua = y la otra diez y seis votixas», Inventario de la estancia de la Sabana del Espíritu Santo, La Sabana del Espíritu Santo, 28 de enero de 1666, ibidem, Mortuoria de Juan de Sologuren, folios 11v-14r.

69 «...tres masas y cinco cobres fundidos que pesan ciento y cincuenta y cinco libras y doce paylas pequeñas sacadas las una por fundición y otra a martillo y un tiesto y una olleta de cobre...», Testamento de Antonio de Reinoso, Mérida, 1 de marzo de 1658, AGEM, Protocolos t. XXIV, folios $10 \mathrm{v}-17 \mathrm{v}$. Igualmente, «...un trapiche a medio uso... más tres masas de bera viexa que están fuerte». Inventario de José de Cepeda y Santa Cruz, San Antonio de Gibraltar, 21 de abril de 1661, Archivo General de la Nación Bogotá (AGNB), Real Audiencia de Bolívar y Venezuela, S.C, 50, 1, Doc. 1. Y, «...una casa de trapiche con un con un moxicón, un par de cadenas de respeto, dos fondos de coser miel, puestos en sus ornallas, uno que hase quinse botijas de agua y otro que hase quatro poco más o menos». Carta de arrendamiento, Torondoy, 4 de diciembre de 1662, AGEM, Asuntos Diversos t. VIII, 1664, n. ${ }^{\circ} 3$, folios $85 \mathrm{r}-87 \mathrm{r}$.

70 «Pesó la payla ocho arrovas que importa a dies reales duzientos y sesenta y dos pesos y quatro reales de los quales se an de revajar beinte pesos y medio que dio Manuel Rodríguez de Andrade al herrero de lo que es, de y lo dio por quenta del capitán Salvador de Trejo y escalfado queda los dichos duzientos y cuarenta pesos y medio del balor de la paila los quales pagaré yo el dicho Manuel Rodríguez de Andrade del asúcar que me remite desde Torondoi del capitán Salvador de Trejo en conformidad de la libranza de arriva y lo firmé», Recibo de pago de una paila de la hacienda de Torondoy, Gibraltar, 15 de noviembre de 1662, AGEM, Asuntos Diversos t. VIII, 1664, n. ${ }^{\circ} 3$, f. 123r.

71 «...tres fondos asentados en las hornallas de cobre batidos, la huna que hiso veinte y ocho votixas de agua = y la otra diez y seis votixas», Inventario de la estancia de la Sabana del Espíritu Santo, La Sabana del Espíritu Santo, 28 de enero de 1666, AGEM, Mortuorias t. VIII, Mortuoria de Juan de Sologuren, folios 11v-14r. 
menea, por la cual se desprendía el humo. En temporada de molienda, se laboraba en los trapiches durante el día y la noche, por lo cual se iluminaban los aposentos con lámparas de fuego cuyo combustible era la grasa de macho $^{72}$.

La zafra se hacía de lunes a viernes, luego se trasladaba la caña al trapiche. El sábado se iniciaba la molienda ${ }^{73}$, se picaba; luego se lavaba y se introducía en las masas, las trituraban en dos ocasiones; la inicial entre el primer y segundo cilindro y otra entre el segundo y tercero, obteniendo dos subproductos, el bagazo, que se acumulaba en la bagacera para combustible y el caldo, que se transportaba a través de canales hasta los fondos para su cocción. Al caldo se le agregaba lejía para que flotara en la superficie la «cachaza», mezcla de desperdicios y mucílagos que se recogían con las agujereadas espumaderas ${ }^{74}$ de cobre, que las retenían en su cuenco, tamizando el líquido.

Después de «descachazado» el caldo, se procedía a colocarlo sucesivamente en tres fondos de cobre hasta que el calor del fuego evaporaba el líquido espesándolo hasta lograr hacer la miel, que se batía agregándole grasa de vaca, cerdo o aceite. Se requerían siete botijas de caldo para obtener una de miel ${ }^{75}$. La miel se comerciaba de tres formas: líquida, templada o procesada para obtener el azúcar. La miel líquida se depositaba en canoas $^{76}$ y luego se envasaba en botijas en las que se transportaba hasta los centros de distribución ${ }^{77}$.

72 «Más dos botijas de manteca de macho para alumbrar el trapiche y el mayordomo a tres pesos cada una montan seis pesos», Libro de cuentas de la hacienda de Torondoy, 1664, AGEM, Asuntos Diversos t. VIII. 1664, n. ${ }^{\circ} 3$, f. 119v.

73 «Más se templó sábado a nuebe de agosto quince panes con la cuenta de seis xarros llenos hasta el gollete cada temple. Más se templo sábado dies y seis de agosto catorce arrobas de asúcar en dies y seis panes. Más se templó sábado a veinte y seis de agosto diez y ocho arrobas de asúcar en veinte y siete panes», Libro de cuentas de la hacienda de Torondoy, 1664, ibidem, f. $175 \mathrm{r}$.

74 «...una espumadera de cobre», Mortuoria de Juan de Sologuren. Inventario de la estancia de la sabana del Espíritu Santo, La sabana del Espíritu Santo, 28 de enero de 1666, AGEM, Mortuorias t. VIII, folios 11v-14r.

75 «...estando uno que son menester siete botijas de caldo para una de miel...», Carta del mayordomo Sebastián Rodríguez a Ignacio Pérez Cerrada, Torondoy, 25 de mayo de 1665,

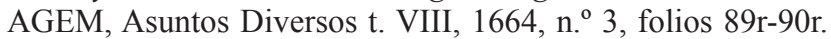

76 «...una canoa adonde se echa la miel con su tapa y candado ya vieja, dice dicho negro que hace cincuenta botixas poco más o menos», Inventario de la hacienda de la Sabana, La Sabana del Espíritu Santo, 30 de septiembre de 1669, AGEM, Mortuorias t. XI Mortuoria de Domingo de Plaza, folios 10v-11r.

77 «...diez y seis botijas de miel como consta de recibo de once de noviembre de mil seiscientos sesenta y cinco, del suso dicho Bartolomé del Castillo = Más otras diez y seis botixas de miel que tiene de recibo del dicho Bartolomé del Castillos de diez y ocho de noviembre de mil seiscientos sesenta y cinco». Inventario de la estancia de la Sabana del Es- 
Existían dos tipos de miel: la buena y la de «purga» o de «barros» ${ }^{78}$ de menor calidad y preción ${ }^{79}$. La miel de «barros», era producto de cañaverales, cuya caña se le calificaba como «ruin», y a partir de la misma era imposible templar panes o elaborar azúcar. Con la miel de purga a los hacendados les era imposible competir con la miel de excelente calidad producida en el valle del Espíritu Santo, en donde se procesaba azúcar óptima, en abundantes cantidades, lo cual hacía descender el precio del producto en el mercado de Gibral$\operatorname{tar}^{80}$. Además, se empleaba la miel para elaborar conservas de membrillos ${ }^{81}$, marañones $^{82}$, limones $^{83}$ y naranjas ${ }^{84}$.

Entre tanto, para obtener panes templados o «panelas» ${ }^{85}$, la miel se depositaba en hormas de madera ${ }^{86}$, de cuya elaboración se encargaba a los indios, que eran diestros en la fabricación de las mismas. Por esa razón, los mayor-

piritu Santo, La Sabana del Espíritu Santo, 28 de enero de 1666, ibidem, t. VIII, Mortuoria de Juan de Sologuren, folios 11v-14r.

78 «Más por el mes de septiembre se ysieron dos botijas de miel de barros que ymbie abajo...», Libro de cuentas de la hacienda de Torondoy, 1665, AGEM, Asuntos Diversos, t. VIII, 1664, n. $^{\circ} 3$, f. 189 r.

79 «...no pongo aquí los del acarreto de la miel porque es al abono puesta en Torondoi a doze reales la miel buena y a seis la miel de purga», Libro de cuentas de la hacienda de Torondoy, 1665, ibidem, f. 192v.

80 «...el cañaveral de la loma que dejó vuestra merced ya echo paja, no se puede aprovechar ni para papelones porque no cuaja, alguna miel se puede hazer colorada que es lo mesmo que nada, respeto de la mucha abundancia que ay de ella, pues casi todos los trapiches se balen de haser asucar y la asen en algunos muy buena con la que a dado de la Sabana, y la que asen los trapiches de lo llano, aunque no sea muy abentajada, se ban a lo barato como lo sabrá vuestra merced de otros y el estado que tiene Gibraltar en razón de plata», Carta de Ignacio Pérez Cerrada a Salvador Trejo de la Parra, Torondoy, 20 de abril de 1663, ibidem, folios 203r-204r.

81 «Más cuarenta membrillos que se cojieron para aser conserva», Libro de cuentas de la hacienda de Torondoy, 1665, ibidem, f. 190r.

82 «Más otra botija de miel que se gastó en conserva de marañones rayada», Libro de cuentas de la hacienda de Torondoy, 1664, ibidem, f. 173r.

83 «...más una botija de miel que se gastó en dos botixas de conserva de lymones», Libro de cuentas de la hacienda de Torondoy, 1665, ibidem, f. 188r.

84 «Más otra botija de miel que se gastó en conserva en naranxa que son dos pesos», Libro de cuentas de la hacienda de Torondoy, 1664, ibidem, f. 173r.

85 «...dos baldes de madera de cargar el caldo, más tres canoas de bera bien tratadas, que son las que sirven con su artesa, dos canoas la una de caldo y la otra para la miel y la una con candado y llave», Inventario de la hacienda de San Antonio de Gibraltar, San Antonio de Gibraltar, 12 de junio de noviembre de 1657, AGEM, Mortuorias t. IV. Mortuoria de Joseph Rodríguez Melo, folios 180r-181r.

86 «...diez y seis hormas». Inventario de la hacienda de la Sabana, La Sabana del Espíritu Santo, 16 de agosto de 1653, ibidem, t. II, Mortuoria de Isabel Cerrada, folios 293r-294r. 
domos les instruían para que cortaran los listones y fabricaran las hormas ${ }^{87}$, en las cuales se dejaba solidificar la panela. Luego se desmoldaban y se empacaban en cascarón de plátano. Tanto en el trapiche de Joseph Rodríguez Melo, ubicado en Chirurí, como en el de doña Isabel Cerrada en el valle de Chama y en el de Salvador Trejo de la Parra en Torondoy, entre otros, se obtenía miel y se solidificaba panela.

La tecnología para producir azúcar era más sofisticada y consistía en depositar la miel en las hormas cónicas de barro ${ }^{88}$, previamente preparadas con dos bocas que reposaban sobre un tendal, con la boca más ancha hacia arriba y la estrecha abajo con un tapón, donde se depositaban varias capas de miel: primero miel menos concentrada, seguida de otra que hubiera engrosado más y luego una de melaza muy densa; sucesivamente se centrifugaban, logrando con ello que la miel que no iba a cristalizar en azúcar se asentara en el fondo de la horma, agregando el «blanquín» o cal para obtener azúcar blanca. Seguidamente, se dejaban reposar durante quince días y después se tapaban las hormas con una delgada capa de arcilla y se dejaba secar por seis días más. Posteriormente, se quitaba el tapón del fondo de la horma dejando drenar la miel que no se había solidificado y se desmoldaba el azúcar para llevarlo al blanqueado. El objetivo final era obtener azúcar blanco pero también se conseguía moscabado ${ }^{89}$, en ocasiones se lograba producir azúcar muy blanco y también los «corazones de azúcar» ${ }^{90}$ que eran el producto más refinado del proceso, los cuales se obtenían en el interior de las hormas.

Era de fundamental importancia en la elaboración del azúcar la pericia de los operarios, porque de lo contrario en manos de inexpertos se corría el riesgo de perder la miel y no lograr el producto con la calidad debida, o en todo caso defectuoso. En ese sentido, las preocupaciones de los mayordomos y administradores fueron mantener a los maestros diestros al frente de las moliendas durante el período de zafra, aunque para lograrlo los trasladaran de

87 «...el yndio cortador que tenga cortada arta leña y se hagan hormas», Carta de Salvador de Trejo a Ignacio de Trejo, Gibraltar, 16 de diciembre de 1662, AGEM, Asuntos Diversos t. VIII, 1664, n. ${ }^{\circ}$ 3. ff, 197r-198v.

88 «...noventa y dos hormas de hacer azúcar de barro vaciadas ítem cincuenta y tres hormas de azúcar en barro», Inventario de la estancia de la Sabana del Espíritu Santo, La Sabana del Espíritu Santo, 28 de enero de 1666, AGEM, Mortuorias t. VIII, Mortuoria de Juan de Sologuren, folios 11v-14r.

89 Ramos Gómez, LXV/233 (Sevilla, 2005): 55.

90 «Más le despaché al amo beinti tres de abril la una botixa de corazones de asúcar con más dos botixas de myel que ymbio a Pedro de Lamo», Libro de cuentas de la hacienda de Torondoy, 1665, AGEM, Asuntos Diversos t. VIII, 1664, n. ${ }^{\circ} 3$, f. 188r. 
una hacienda a otra, exigencia que se hacía con premura, pero garantizando que los retornarían a sus residencias ${ }^{91}$.

El azúcar se despachaba al puerto en panes de azúcar que pesaban los grandes una arroba, y los pequeños media, con diferente valor ${ }^{92}$, transportados sobre mulas cuya adquisición como de las enjalmas y petacas ${ }^{93}$ o cestas donde se trasladaban los productos, las cuales fueron una constante preocupación de los mayordomos, además la eficiencia en su transporte, para evitar pérdidas ${ }^{94}$.

\section{LAS CUENTAS DE LA HACIENDA AZUCARERA EN TORONDOY}

El proceso productivo cañamelero en el sur de lago de Maracaibo, se puede apreciar en la hacienda del capitán Salvador Trejo de la Parra. El predio fue parte de las extensas tierras que fueron mercedadas a su abuelo Miguel Trejo de la Parra en la segunda mitad del siglo XVI, y Salvador la obtuvo como parte de la herencia de su padre, también llamado Miguel Trejo, ubica-

91 «Esta semana e molido como una payla porque la otra no e tenido con que taparla, oy templé para hacer la prueba media docena de panes de asúcar no se que tal saldrán, porque no estoy muy diestro en ello, falta nos hace el no aver beinido el yndio Simonico y siempre tube librada las esperansas en que vuestra merced me lo remitiría. Ay embio al cacique a que me lo traiga y podrá vuestra merced el fabor de que lo consiga que le aseguro de aserle ningún mal pasaje, sino que este muy contento. Y en acabado de haser el asúcar le daré permiso que se vuelva, mucho nos ymportara que benga porque ya es poca la asúcar que ay que moler este año, no arriesguemos a hecharla a perder», Carta de Salvador de Trejo a Ignacio de Trejo, Torondoy, 3 de marzo de 1663, ibidem, folios 199r-200v.

92 «...le despaché a Juan de Baldibiesso por le mes de abril beynte y quatro panes de asúcar que pesaron veinticinco arrobas y dies y seis libras de asúcar. 27. Más le despaché al dicho Juan de Baldibiesso a principios del mes de mayo del año de sesenta y tres beinte y seis panes de asúcar que pesaron beintisiete arrobas y ocho libras», Libro de cuentas de la hacienda de Torondoy, 1664, ibidem, f. 116r.

93 «...y allí servirse vuestra merced de enviarme uno o dos pares de petacas usadas, porque no las tengo para acá a quien sacar el asúcar y envíeme con ellas once arrobas de lona», Carta de Salvador de Trejo a Ignacio de Trejo, Torondoy, 1 de mayo de 1663, ibidem, folios 205r-206r.

94 «...y si las mulas no se ubiere despachado el corregidor, sírvase vuestra merced de que luego que llegue imbiermelas con el yndio Juan y que se le entreguen a Marquitos y que no dé bestia ninguna del Sr. Gobernador, sino es llevando letra de su merced. A Dominguito dígale vuestra merced que otro día luego después de pascua me embie las tres mulas mías, que están allá en el potrero, con la enjalma que llevó ayer Nicolasito i dos aparejos que acá tengo y otra enjalma a este Francisco», Carta de Salvador de Trejo a Ignacio de Trejo. Torondoy, 15 de junio de 1663, ibidem, folios 201r-202v. 
do en el sitio de Mocoguas o como la denomina su propietario en Mucuguay95, circundada por los ríos Torondoy y Capio. La propiedad estaba cultivada con cañaverales, tenía edificaciones para la vivienda del mayordomo y los trabajadores, un trapiche con tracción de sangre, además tenía huertas cultivadas con repollos y membrillos, los cuales también se remitían al puerto para su comercialización.

El manejo de la hacienda estaba confiado a los mayordomos, evidentemente los hubo más experimentados y otros menos diestros y esa cualidad determinó el rendimiento de la producción de azúcar, miel, y conservas. El azúcar se elaboraba en panes que se empacaban en dos presentaciones: los grandes, cuyo peso se estimaba en una arroba de 12 kilogramos o 24 libras, y pequeños contentivos de media arroba, estimado en 6 kilogramos o 12 libras. Mientras, la miel se envasaba en botijas.

Los libros de cuentas y gastos llevados por Sebastián Rodríguez e Ignacio Pérez Cerrada corresponden a 1664-1667. A través de esos registros contables se puede precisar que la producción de la hacienda fue mayoritariamente de miel buena con 26 botijas, mientras la miel de purga se reservó para la elaboración de conservas, tanto en la misma hacienda o en las vecinas. Mientras del azúcar refinado, se produjeron 504 arrobas y 18 libras, enviadas al puerto en 530 panes. El tercer producto obtenido fue el de templar papelones o panelas, de las que se produjeron 5, cuyo peso se estimó en 39 arrobas y 18 libras. (Véase tabla 1).

TABla 1. Producción de MiEl, AZÚCAR y PANELA EN LA HACIENDA DE TORONDOY. 1664-1667

\begin{tabular}{|c|c|c|c|c|c|c|c|}
\hline \multirow{2}{*}{ Años } & $\begin{array}{c}\text { miel } \\
\text { (botijas) }\end{array}$ & \multicolumn{2}{|c|}{ azúcar } & panes & \multicolumn{3}{c|}{ papelones } \\
\cline { 2 - 8 } & unidad & $@$ & $\&$ & unidad & $\begin{array}{c}\text { Can- } \\
\text { tidad }\end{array}$ & a & \& \\
\hline 1664 & 26 & 183 & 11 & 198 & 5 & 39 & 18 \\
\hline 1665 & & 213 & & 248 & & & \\
\hline 1666 & & 87 & & 84 & & & \\
\hline 1667 & & 21 & 7 & & & & \\
\hline total & 26 & 504 & 18 & 530 & 5 & 39 & 18 \\
\hline
\end{tabular}

Fuente: AGEM, Asuntos Diversos, t. VIII, 1664, 3.

95 Testamento de Miguel de Trejo. Mérida, [sin fecha] 1642, AGEM, Protocolos t. XVII, folios $239 \mathrm{r}-245 \mathrm{v}$. 
El azúcar estuvo dirigida a distintos destinatarios, se reservó al consumo de la hacienda, 28 arrobas. Además, se enviaron al corregidor de naturales 2 arrobas y 20 libras, mientras a indios, pardos e inclusive mulatos, se les vendieron 6 y 38 libras. También, se reservaron panes de azúcar para el pago de diezmos ${ }^{96}$ y los estipendios a los doctrineros de Torondoy ${ }^{97}$, destinándose 3 arrobas y 18 libras. La mayor cantidad de la producción de azúcar se consignó al arrendatario en 341 arrobas y 184 libras y se enviaron al puerto 296 arrobas y 183 libras para su mercantilización. Obviamente, la mayor cantidad de azúcar se destinaba a los expendedores que tenían sus tiendas en Gibraltar. Además, se procesaron 4 arrobas y 33 libras en conservas de limones naranjas y membrillos. (Véase tabla 2)

TABla 2. Distribución DEL AZÚCAR PRODUCIDO EN LA HACIENDA DE

TORONDOY. 1664-1667

\begin{tabular}{|c|c|c|c|c|c|c|c|c|c|c|c|c|c|c|c|c|}
\hline \multirow{2}{*}{ año } & \multicolumn{2}{|c|}{ Gasto } & \multicolumn{2}{|c|}{ Corre-gidor } & \multicolumn{2}{|c|}{$\mathrm{Al}$ amo } & \multicolumn{2}{|c|}{ Otros } & \multicolumn{2}{|c|}{ Religiosos } & \multicolumn{2}{|c|}{ Puerto } & \multicolumn{2}{|c|}{ Conserva } & \multicolumn{2}{|c|}{$\begin{array}{c}\text { Sin } \\
\text { especificar }\end{array}$} \\
\hline & (a) & $\&$ & (a) & $\&$ & (a) & $\&$ & (a) & $\&$ & (a) & $\&$ & (a) & $\&$ & (a) & $\&$ & (a) & $\&$ \\
\hline 1664 & 4 & & 2 & 20 & 189 & 111 & 1 & 12 & 4 & 36 & & & 4 & 24 & & \\
\hline 1665 & 24 & & & & 78 & 31 & & & 10 & 37 & 296 & 183 & & 21 & & \\
\hline 1666 & & & & & 74 & 44 & 5 & 26 & 3 & 18 & & & & 33 & & \\
\hline 1667 & & & & & & & & & & & & & & & 21 & 7 \\
\hline total & 28 & & 2 & 20 & 341 & 184 & 6 & 38 & 17 & 91 & 296 & 183 & 4 & 78 & 21 & 7 \\
\hline
\end{tabular}

Fuente: AGEM, Asuntos Diversos, t. VIII, 1664, 3.

Los ingresos estuvieron determinados por diferentes eventos que afectaron el proceso productivo. Durante los años que se produjo en condiciones normales rindieron después de su expendio entre 1.156 y 1.286 pesos anuales. Pero, a diferencia de ese lapso, en 1666, la molienda de la caña se inició en

96 «Y de esta cuenta y demás cosas que se hicieron asta el año de sesenta y cinco pagué de diesmo a don Juan de Monasterio a quien le pertenecía doce @ de asúcar, una votija de miel, setenta membrillos y dies pesos en plata, por lo demás en quenta me conserté vine con el dicho como consta por recibo suyo». En otro asiento se expresa lo siguiente: «...pagué de diesmo nueve@ @e asúcar a Juan Martín de Velasco y lo que tocó de miel y demás cosas pagué en dinero, componiéndome con dicho dinero como consta de su recibo firmado de su nombre a dos de febrero de del año de sesenta y sinco», Ajuste de cuentas, San Antonio de Gibraltar, 22 de diciembre de 1667, AGEM, Asuntos Diversos t. VIII, 1664, n. ${ }^{\circ}$ 3, f. 212r.

97 «Más a dos de agosto se le dieron otras dos votijas de miel al doctrinero», Ajuste de cuentas, San Antonio de Gibraltar, 22 de diciembre de 1667, ibidem, f. 215 r. 
junio, y un mes más tarde ocurrieron los infaustos sucesos ocasionados por la invasión del pirata el Olonés, cuyo evento detuvo la siega y se perdieron los cañaverales ${ }^{98}$. Otro factor negativo que se enfrentó en el proceso productivo concurrente con el anterior fue la negativa a laborar de los indígenas. Los naturales se rehusaron a acudir al beneficio de la hacienda debido al retraso en la confirmación del título de encomienda a Salvador de Trejo. Esa situación se debió a que se había planteado un pleito por su sucesión ${ }^{99}$. Debido a esas adversas circunstancias, tanto la producción como el ingreso cayeron a 499 pesos, debido a que en noviembre se reinició el proceso de zafra. Finalmente, está el año de 1667, solo enero y febrero, rindieron 130 pesos. En total se produjeron 3.072 pesos (Véase tabla 3).

\section{TABla 3. Ingresos BRUtos de LA HACIENDA DE TORONDOY.} $1664-1667$

\begin{tabular}{|c|c|c|}
\hline año & Pesos & Reales \\
\hline 1664 & 1156 & 1 \\
\hline 1665 & 1286 & 6 \\
\hline 1666 & 499 & 3 \\
\hline 1667 & 130 & 4 \\
\hline total & 3072 & 6 \\
\hline
\end{tabular}

Fuente: AGEM, Asuntos Diversos, t. VIII, 1664, 3.

Los egresos de la hacienda estuvieron representados por la satisfacción de diferentes necesidades inherentes al proceso productivo agrario. En primer término, se destacan los salarios que se pagaban al mayordomo, a razón de 120 pesos anuales. A esto, es necesario agregar los que se saldaban a los

98 «... no se comenzó a moler hasta principio del mes de junio de dicho año, en que aviéndose comensado dicha molienda, llegó luego a principios del mes de julio la nueva de la entrada del enemigo por la varra, con que se embarasso y atrasó todo como es público», Ajuste de cuentas, San Antonio de Gibraltar, 22 de diciembre de 1667, ibidem, f. 216r.

$99 \ll .$. y no se pudo de allí adelante moler cosa de fundamento porque los yndios no acudían al veneficio de dicha hacienda, así por aver faltado mi asistensia en ella, como porque también llegó a su noticia la restitución que la Real Audiencia hiso al capitán Salvador de Trexo de la dicha encomienda de ellos: con que mediante esta noticia que se adquirieron no quisieron acudir a derechas al veneficio...», Ajuste de cuentas, San Antonio de Gibraltar, 22 de diciembre de 1667 , ibidem, n. ${ }^{\circ} 3$, f. 216 r. 
maestros carpinteros, por las refacciones del trapiche, a quienes se liquidaron 20 pesos. En total se pagaron por jornales 420 pesos. (Véase tabla 4)

Tabla 4. Egresos en la hacienda De Torondoy

$1664-1667$

\begin{tabular}{|l|r|r|r|r|r|}
\hline \multicolumn{1}{|c|}{ conceptos } & $\mathbf{1 6 6 4}$ & $\mathbf{1 6 6 5}$ & $\mathbf{1 6 6 6}$ & $\mathbf{1 6 6 7}$ & Total \\
\hline salarios & 140 & 120 & 160 & & 420 \\
\hline carne & 28 & 24 & 22 & & 74 \\
\hline manteca & 6 & 6 & 6 & & 18 \\
\hline pescado & 9 & 8 & 11 & & 28 \\
\hline queso & & 4 & 2 & & 6 \\
\hline cacao & & 12 & 9 & & 21 \\
\hline sal & 9 & 8 & 6 & & 23 \\
\hline manteca de macho & 9 & 3 & 7 & & 19 \\
\hline jabón & 2 & 2 & & & 4 \\
\hline herramientas y refacciones & 10 & 14 & 10 & & 34 \\
\hline fletes & 64 & 72 & 31 & & 167 \\
\hline bueyes & & & & 30 & 30 \\
\hline petacas & & & & 10 & 10 \\
\hline caballos & 277 & 273 & 264 & 60 & 874 \\
\hline totales & & & & 20 & 20 \\
\hline
\end{tabular}

Fuente: AGEM, Asuntos Diversos, t. VIII, 1664, 3.

En segundo término la adquisición de alimentos. En este rubro existieron variaciones debido al incremento normal de los costos de los insumos, especialmente en los períodos difíciles, producto de la invasión del Olonés, que determinó desabastecimiento de comestibles y que se elevaran sus costos. El primer rubro reseñado es la carne, cuyo valor oscilaba entre 12 reales y 16 reales la arroba, se gastaron entre 22 y 28 pesos anuales, para un total de 74 pesos. Otros rubros fueron la manteca para el consumo humano, en la cual se invirtieron 18 pesos; el pescado, cuyo costo se elevó a 28 pesos; el queso a 6 pesos y el cacao a 21 pesos. La sal se adquiría para consumo humano y animal, cuyo costo fue 23 pesos. Mientras, se invirtieron 19 pesos en la manteca de macho, destinada al alumbrado del trapiche y las casas de la hacienda. Asimismo, 4 pesos en el jabón empleado en el consumo humano y del trapiche. Además, 34 pesos invertidos en una reja para el arado y las refacciones para «aliñar» el trapiche, sus cadenas y goznes. Y en la adquisición de las 
pailas para la cocción del caldo de caña. (Véase tabla 4), se invirtieron 30 pesos. Mientras, los caballos moledores costaron 20 pesos y las petacas o cestos importaron 10 pesos. En total durante el lapso estudiado se invirtieron 874 pesos en el gasto de funcionamiento de la hacienda (Véase tabla 4).

El balance de la hacienda de Torondoy presenta los siguientes resultados: en 1664, la hacienda produjo 1.156 pesos y 1 real, mientras los egresos fueron 277 pesos, lo que aportó una renta neta de 877 pesos. En el año siguiente, es decir 1665 , los ingresos se elevaron a 1.286 pesos y 6 reales mientras que los egresos se mantuvieron casi al mismo nivel del año anterior en 273 pesos, proporcionando una ganancia de 1.013 pesos y 6 reales. Por el contrario, en 1666 , en un año del asalto pirático, los ingresos descendieron a 499 pesos y 3 reales, manteniendo el nivel de egresos en 264 pesos, y por lo tanto reduciendo la rentabilidad de la hacienda a la irrisoria cantidad de 235 pesos y 3 reales. Entre tanto, en los dos meses del año de 1667, ingresaron 130 pesos y se gastaron 60, obteniendo un margen de ganancia de 70 pesos y 4 reales. Los ingresos se elevaron a 3.072 pesos y 6 reales, mientras los egresos se cuantificaron en 874 pesos. Esas cifras permiten apreciar que la renta de la hacienda fue de 2.198 pesos y seis reales, lo cual revela las importantes ganancias que se obtenían en aquellos núcleos de producción (Véase tabla 5).

Tabla 5. Balance de ingresos y gastos en la hacienda Torondoy. $1664-1667$

\begin{tabular}{|c|c|r|r|r|r|}
\hline \multirow{2}{*}{ año } & \multicolumn{2}{|c|}{ ingresos } & egresos & \multicolumn{2}{c|}{ renta } \\
\cline { 2 - 6 } & $\$$ & Rs. & $\$$ & $\$$ & Rs. \\
\hline 1664 & 1156 & 1 & 277 & 879 & 1 \\
\hline 1665 & 1286 & 6 & 273 & 1013 & 6 \\
\hline 1666 & 499 & 3 & 264 & 235 & 3 \\
\hline 1667 & 130 & 4 & 60 & 70 & 4 \\
\hline total & 3072 & 6 & 874 & 2198 & 6 \\
\hline
\end{tabular}

Fuente: AGEM, Asuntos Diversos, t. VIII, 1664, 3.

\section{Conclusiones}

La creciente actividad económica, desarrollada en la planicie del sur del Lago de Maracaibo, tuvo una rápida expansión. Fueron de significativa importancia las estancias productoras de caña de azúcar, que rápidamente evolucionaron hacia haciendas, asentadas en una rápida expansión de la frontera 
agrícola, lo que determinó el inusitado aumento de la producción de miel, panela, melotes y azúcar. En consecuencia, las haciendas cañameleras en la planicie lacustre definieron con precisión sus sistemas productivos sobre la base de la eliminación total de los sotobosques para ser sustituidos por los cultivos de caña de azúcar. Del mismo modo, la implantación de la tecnología tanto en el cultivo de la caña como en su procesamiento, mediante la introducción de herramientas e ingenios provenientes de las Antillas, que fueron modificadas y adaptadas a las necesidades particulares del área. Por otra parte, la infraestructura de las haciendas estuvo integrada por un complejo que comprendía casa y trapiche.

\section{BiBLIOGRAFÍA}

Academia Nacional de la Historia, "Relación Geográfica hecha por Diego de Villanueva y Gibaja de la Gobernación de Venezuela, los Corregimientos de La Grita y de Tunja y la Gobernación de los Mussos. Año de (1607)", Relaciones geográficas de Venezuela, recopilación, estudio preliminar y notas de Antonio Arellano Moreno, Caracas, Academia Nacional de la Historia, 1964. Colección Fuentes para la Historia Colonial de Venezuela, 70.

Andrein, Kenneth J., "The sale of juros and the politics of reforms in the Viceroyalty of Peru", Journal of Latin American Studies, 13/1 (Cambridge, may 1981): 1-19.

Arcila Farías, Eduardo; Maza Zavala, D. F.; Brito Figueroa, Federico y Tovar, Ramón A., La obra pía de Chuao. 1569-1825, Caracas, Comisión de Historia de la propiedad agraria territorial/Universidad Central de Venezuela, 1968, t. 1.

Arango Estrada, Vicente Fernán, La endogamia en las concesiones antioqueñas, Manizales, Hoyo Editores, 2003.

Banko, Catalina, "La industria azucarera en Venezuela y México. Un estudio comparativo", Boletín de la Academia Nacional de la Historia, LXXXVIII/352 (Caracas, octubre-diciembre 2005a): 157-179.

Banko, Catalina, "Proceso de modernización, auge y estancamiento de la agroindustria azucarera en Venezuela", Tierra Firme, 91/23/XXIII (Caracas, 2005b): 341-360.

Barret, Ward, The Sugar Hacienda of the Marqueses del Valle, Minneapolis, University Press, 1970.

Barret Ward J. y Schwartz Stuart, B., "Comparación entre dos economías azucareras coloniales: Morelos, México y Bahía, Brasil”, Enrique Florescano (eds.), Haciendas, latifundios y plantaciones en América Latina, México, SIAP-CLACSO/Siglo XXI Editores, 1979: 532-571. 
Bauer, Arnold, "The church in the economy of Hispanic American. Censos and depósitos in eighteenth and nineteenth centuries", Hispanic American Historical Review, 63/4 (Duke, November 1983): 711-740.

Bauer, Arnold (ed.), La Iglesia en la economía de América Latina siglos XVI al XIX, México, INAH, 1986.

Bazant, Jan, Cinco haciendas mexicanas. Tres siglos de vida rural en San Luis Potosí, 1600-1910, México, El Colegio de México, 1975.

Borah, Woodrow, New Spain Century of Depression, Berkeley, University of California Press, 1951.

Birocco, Carlos María, "Historia de un latifundio rioplatense: las estancias de Riblos en Areco. 1713-1813", Anuario de Estudios Americanos, LIII/1 (Sevilla, 1996): 7399.

Brading, David, Estructura de la producción agrícola en el Bajío, 1700-1850, Enrique Florescano (ed.), Haciendas, latifundios y plantaciones en América Latina, México, SIAP-CLACSO/Siglo XXI Editores, 1979: 49-69.

Chevalier, François, La Formation des Grands Domaines en Mexique. Terre et Société aux XVI e XVII Siècles, Paris, Institut d'Ethnologie, 1952.

Colmenares, Germán, Las haciendas jesuitas en el Nuevo Reino de Granada, Bogotá, Universidad Nacional de Colombia, 1969.

Colmenares, Germán, Historia económica y social de Colombia. 1537-1719, Bogotá, Lealón, 1977.

Colmenares, Germán, Historia económica y social de Colombia. (Popayán: una sociedad esclavista. 1680-1800), Bogotá, Editorial La Carreta, 1979.

Criton, Michael, "Worthy Park. 1670-1972. Cambios y continuaciones del sistema jamaiquino de plantación azucarera", Enrique Florescano (ed.), Haciendas, latifundios y plantaciones en América Latina, México, SIAP-CLACSO/Siglo XXI Editores, 1979: 573-608.

Escorcia, José, "Haciendas y estructura agraria en el valle del Cauca", Anuario Colombiano de Historia Social y de la Cultura, 10 (Bogotá, 1982): 119-137.

Espinoza, Andrés Benito, La hacienda Chichuy 1558-1800, Mérida, Venezuela, Universidad de Los Andes (tesis), 1980.

Ferreira, Esparza y Adriana, Carmen, “Capellanías y censos. Una conceptualización necesaria para el estudio del crédito colonial”, Ensayos de historia regional de Santander, Bucaramanga, Universidad Tecnológica Experimental de Santander, 1995: $38-78$.

Follet, Richard, "Give to the Labor of America, The Market of America. Marketing. The Old South's Sugar Crops”, Revista de Indias, LXV/233 (Madrid, 2005): 117-146. 
Franklin, Raúl (ed.), La historia agraria en el Río de la Plata colonial. Los establecimientos productivos, Buenos Aires, Centro Editor de América Latina, 1992, t. I y II.

Gelamby de Montilla, Darcy, La hacienda los Curos, Mérida, Venezuela, Universidad de Los Andes (tesis), 1979.

García Muñiz, Humberto, "La plantación que no se repite: Las historias azucareras de la República Dominicana y Puerto Rico, 1870-1930", Revista de Indias, LXV/233 (Madrid, 2005): 173-192.

González Sánchez, Isabel, Haciendas y ranchos en Tlaxcala en 1712, México, Instituto Nacional de Antropología e Historia, 1969.

Jackson, Robert H. y Gordillo, Claure José, "Formación, crisis y transformación de la estructura agraria de Cochabamba. El caso de la hacienda de Paucarpata y de la comunidad del Passo. 1538-1645 y 1872-1929”, Revista de Indias LIII/199 (Madrid, 1993): 723-759.

Jiménez Pelayo, Águeda, "El impacto del crédito en la economía rural de Nueva Galicia", The Hispanic American Historical Review, 71/3 (Duke, August 1991): 501-529.

Klaren, Peter F., “The Sugar Industry en Perú”, Revista de Indias, LXV/233 (Madrid, 2005): 33-48.

Kicza, John E., Empresarios coloniales. Familias y negocios en la ciudad de México durante los Borbones, México, Fondo de Cultura Económica, 1986.

Konetzke, Richard, América Latina II. La época colonial, México, Siglo XXI Editores, 1977. Colección Historia Universal Siglo XXI, 22.

Ladd, Doris M., La nobleza mexicana en la época de la Independencia, 1780-1826, México, Fondo de Cultura Económica, 1984: 39-40.

Langer, Eric D., y Hames, Gina, "Commerce and credit on the periphery: Tarija merchants. 1830-1841", Hispanic American Historical Review, 74/2 (Duke, may 1994): 285-301.

Langue, Frédérique, Aristócratas, honor y subversión en la Venezuela del siglo XVIII, Caracas, Academia Nacional de la Historia, 2000. Colección Fuentes para la Historia Colonial de Venezuela, 252.

Lavrin, Asunción, "The role of the nunneries in the economy of the New Spain in the eighteenth century", The Hispanic American Historical Review, XLVI/4 (Duke, November 1966): 372-393.

Lavrin, Asunción, "The execution of the Law of Consolidación in New Spain Economic Aims and Results", Hispanic American Historical Review, 53/1 (Duke, February 1993): 27-49.

Lavrin, Asunción, "El Convento de Santa Clara de Querétaro. La Administración de sus propiedades en el siglo XVII", Revista Historia Mexicana, XXV/97 (México, julio-noviembre 1975): 75-116. 
Linder, Peter S., "Relaciones de producción en las haciendas del sur del lago zuliano, 1880-1936. Algunas conclusiones preliminares", Tierra Firme, 19/V/5 (Caracas, julio-septiembre 1987): 283-291.

Lindley, Richard B., Las haciendas y el desarrollo económico, México, Fondo de Cultura Económica, 1987.

Lockhart, James, El mundo hispanoperuano. 1532-1560, México, Fondo de Cultura Económica, 1982.

Macera, Pablo, "Feudalismo colonial americano. El caso de las haciendas peruanas", Acta Histórica, XXX/IV (Szeccd, Hungría, 1971): 3-43.

Marmolejo Salazar, Beatriz, Tablante Blanca, El censo fuente generadora de crédito: su evolución histórica y la participación de la Iglesia como agente crediticio, particularidades en Mérida. 1785-1800, Mérida, Venezuela, Universidad de Los Andes (tesis), 1984.

Martínez Calvo, María del Pilar (ed.), Iglesia, Estado y economía. Siglos XVI al XIX, México, Instituto de Investigaciones Históricas de la Universidad Nacional Autónoma de México, 1995.

Maza Zavala, Domingo F., "La estructura económica de una plantación colonial en Venezuela”, Eduardo Arcila Farías, D. F. Maza Zavala, Federico Brito Figueroa y Ramón A. Tovar (eds.), La Obra Pía de Chuao. 1568-1825, Caracas, Comisión de historia de la propiedad territorial y agraria de Venezuela/Ediciones de la Universidad Central de Venezuela, Instituto de Estudios Hispanoamericanos, 1968, tomo 1: 51-112.

Millares Carlo, Agustín, Protocolos del Siglo XVI, Caracas, Academia Nacional de la Historia, 1966. Colección Fuentes para la Historia Colonial de Venezuela, 80.

Molina, Luis E., "Historia y arqueología de un antiguo ingenio azucarero en el valle de Caracas, Venezuela”, Annales del Museo de América, 13 (Caracas, 2005): 199-224.

Moreno Fraginals, Manuel: El ingenio complejo económico social cubano. El azúcar, La Habana, Editorial de Ciencias Sociales, 1978, 3 vols.

Mörner, Magnus, "En torno a las haciendas en la región del Cuzco desde el siglo XVIII”, Enrique Florescano (ed.), Haciendas, latifundios y plantaciones en América Latina, México, SIAP-CLACSO/Siglo XXI Editores, 1979: 316-396.

Quiroz, Alfonso W., "Reassessing. The role of credit in the late colonial Peru: Censos, escrituras, and imposiciones”, Hispanic American Historical Review, 1/50 (Duke, 1994): 194-229.

Ramos Gómez, Oscar Gerardo, "Caña de Azúcar en Colombia”, Revista de Indias, LXV/233 (Madrid, 2005): 49-78. 
Ramírez Méndez, Luis Alberto, De la piedad a la riqueza. El convento de Santa Clara de Mérida. 1651-1874, Mérida, Venezuela, Archivo Arquidiocesano de Mérida, 2005, tomo I. Colección Fuentes para la Historia Eclesiástica de Venezuela, 4.

Riley, G. Michael, "El prototipo de la hacienda en el centro de México. Un caso del siglo XVI", E. Florescano (ed.), Haciendas, latifundios y plantaciones en América Latina, México, SIAP-CLACSO/Siglo XXI Editores, 1979: 49-69.

Riley, James Denson, "Santa Lucía: Desarrollo y administración de una hacienda jesuita en el siglo XVIII", E. Florescano (ed.), Haciendas, latifundios y plantaciones en América Latina, México, SIAP-CLACSO/Siglo XXI Editores, 1979: 242-272.

Rodríguez, Marisol, Cuando llovió azúcar en Bobures... La industria azucarera zuliana, génesis del empresariado venezolano. 1890-1940, Maracaibo, Ediciones del ViceRectorado Académico de la Universidad del Zulia, 2008. Colección Textos Universitarios.

Ruiz Tirado, Mercedes, Tabaco y sociedad en Barinas siglo XVII, Mérida, Venezuela, Universidad de Los Andes, 2000.

Taylor, William, "Haciendas coloniales en el valle de Oaxaca", Enrique Florescano (ed.), Haciendas, latifundios y plantaciones en América Latina, México, SIAP-CLACSO/Siglo XXI Editores, 1979: 71-102.

Tovar Pinzón, Hermes, "Elementos constitutivos de la empresa agraria jesuita en la segunda mitad del siglo XVIII en México", E. Florescano (ed.), Haciendas, latifundios y plantaciones en América Latina, México, SIAP-CLACSO/Siglo XXI Editores, 1979: 132-241.

Tovar Pinzón, Hermes, Colombia imágenes de su diversidad (1492 a Hoy), Bogotá, Grupo Editorial Educar, 2007.

Sánchez Román, José Antonio, "La industria azucarera en Argentina (1860-1914) El mercado interno en una economía exportadora”, Revista de Indias, LXV/233 (Madrid, 2005): 147-172.

Santamaría García, Antonio y García Álvarez, Alejandro, "Azúcar: en América”, Revista de Indias, LXV/233 (Madrid, 2005): 9-32.

Samudio A., Edda O., Las haciendas del colegio San Francisco Xavier de la Compañía de Jesús en Mérida. 1628-1767, Caracas, Universidad de Los Andes/Editorial Arte, 1985.

Samudio A., Edda O., "Los esclavos de las haciendas del Colegio de San Francisco Javier de Mérida", Paramillo, 17 (Caracas, 1998): 451-548.

Samudio A., Edda O., "Las haciendas jesuíticas de las misiones de los llanos del Casanare, Meta y Orinoco", Misiones Jesuíticas de la Orinoquia, San Cristóbal (Venezuela), Universidad Católica del Táchira, 1993, t, I, 
Samudio A., Edda O., El colegio San Francisco Javier en la Mérida colonial. Germen histórico de la Universidad de Los Andes, Mérida, Ediciones de la Universidad de Los Andes, 2003. t. I.

Sandoval, Fernando B., La industria azucarera en Nueva España, México, Instituto de Historia, Universidad Nacional Autónoma de México, 1951.

Schwartz, Stuart B., "A Commonwealth within Itself. The Early Brazilian Sugar Industry, 1550-1670”. Revista de Indias, LXV/233 (Madrid, 2005): 79-116.

Siegrist, Nora y Samudio A., Edda O. (eds.), Dote matrimonial y redes de poder en el Antiguo Régimen en España e Hispanoamérica, Mérida, Venezuela, Universidad de Los Andes, 2006.

Socolow, Susan, “Cónyuges aceptables. La elección del consorte en Argentina colonial. 1778-1810", Asunción Lavrin (ed.), Sexualidad y matrimonio en la América hispana, México, Grijalbo, 1991: 229-270.

Suárez de Paredes, Niria, Apuntes para el estudio de la hacienda andina del siglo XIX: el caso de la hacienda de la Santísima Trinidad de Los Curos, 1880-1884, Mérida, Venezuela, Universidad de Los Andes, 1984.

Suárez de Paredes, Niria, Formación histórica del sistema cañamelero. 1600-1900, Mérida, Venezuela, Archivo Arquidiocesano de Mérida, 2001. Serie Estudios, 2.

Stanley, J. y Stein, Bárbara H., La herencia colonial de América Latina, México, Siglo XXI Editores, 1975.

Tallaferro, Julio César, La hacienda Estanques 1721-1877, Apuntes para su historia, Mérida, Venezuela, Universidad de Los Andes, 1979.

Tavera Marcano, Carlos Julio, Historia de la propiedad territorial en el valle de Aragua, Maracay, Venezuela, Gobernación del Estado Aragua/Academia Nacional de la Historia, 1995.

Terán Najas, Rosemarie, "Censos, capellanías y élites", Procesos. Revista Ecuatoriana de Historia, II Semestre/1 (Quito, 1991): 22-48.

Torres Castro, Edgar A., "Participación de las estancias en las economías locales y regionales: El caso de Susa en el Nuevo Reino de Granada. Siglo XVII", Diana Bonet Vélez, Michael de la Rosa, Germán R. Mejía Pavony y Mauricio Nieto Olarte (eds.), Nueva Granada colonial. Selección de textos históricos, Bogotá, Universidad de Los Andes, 2003: 227-250.

Troconis de Veracoechea, Ermila, La obras pías en la Iglesia colonial venezolana, Caracas, Academia Nacional de la Historia, 1971. Colección Fuentes para la Historia Colonial de Venezuela, 105.

Troconis de Veracoechea, Ermila, Los censos en la Iglesia Colonial Venezolana (Sistema de préstamos a interés), Caracas, Academia Nacional de la Historia, 1982, t. I. Colección Fuentes para la Historia Colonial de Venezuela, 153. 
Von Wobeser, Gisela, El crédito en Nueva España, México, UNAM, 1994.

Villamarín, Juan A., "Haciendas en la sabana de Bogotá, Colombia en la época colonial, 1539-1810", E. Florescano (ed.): Haciendas, latifundios y plantaciones en América Latina, México, SIAP-CLACSO/Siglo XXI Editores, 1979: 145-160.

Wolf, Erick R. y Mintz, Sydney W., "Haciendas y plantaciones en Mesoamérica y las Antillas", E. Florescano (ed.), Haciendas, latifundios y plantaciones en América Latina, México, SIAP-CLACSO/Siglo XXI Editores, 1979: 493-591.

Fecha de recepción: 4 de abril de 2012.

Fecha de aceptación: 4 de julio de 2012.

\section{Sugar plantations south Maracaibo lake-Venezuela (centurys XVI-XVII)}

This analysis studies sugar cane plantations located south of Lake Maracaibo, in the jurisdiction of Merida (Venezuela), where sugar cane was cultivated and processed from the sixteenth century. It is a quantitative and qualitative study of the origin, evolution, production, infrastructure, and profitability of the plantations. The research is based on unpublished documentary sources in national and foreign archives.

KEY WORDS: sugar cane plantations; sugar mill; honey; molasses; sugar. 